\section{Interruptions during hospital nurses' medication administration rounds}

\author{
Marian Smeulers, ${ }^{1}$ Marjoke Hoekstra, ${ }^{2}$ \\ Emma van Dijk, ${ }^{2}$ Femke Overkamp, ${ }^{2}$ \\ Hester Vermeulen ${ }^{1,2}$ \\ 'Department of Quality and Process \\ Innovation, Academic Medical Center; \\ 2Department of Nursing, Amsterdam \\ School of Health Professions, \\ Amsterdam, The Netherlands
}

\section{Abstract}

Medication administration errors are common, costly and the cause of adverse events in clinical practice. Interruptions during medication administration rounds are thought to be a prominent causative factor of these medication errors. In this observational study, data were collected on the number and duration of several different sources of verbal and non-verbal interruptions using unobtrusive structured observations on 32 medication administration rounds. Interruptions occurred very often (6.9 times per nurse each hour), differed in frequency among the medication administration rounds and were from a variety of sources. The most frequent interruptions were caused by nursing colleagues (43\%) and non-verbal interruptions from the ward environment (25\%), such as noises from pagers, conversations in the vicinity of the nurse, the work of cleaners, or stock management by pharmacy staff. The longest durations of interruptions were from nursing colleagues' verbal interruptions. When comparing the medication rounds, more and longer interruptions were observed during the morning rounds than those at noon. A comparison between surgical and non-surgical units showed that interruptions occurred more often and lasted longer in non-surgical units than those in surgical units. But the observed differences were not statistically significant. In conclusion, interruptions during medication administration rounds are frequent and originated from different human and environmental sources. Interventions should target not only interruptions by colleagues, but should also consider ways to reduce self-initiated interruptions and those arising from the immediate ward environment.

\section{Introduction}

The most common type of errors and pre- ventable cause of adverse events is medication errors (MEs). ${ }^{1}$ An ME is defined as any preventable event that may cause or lead to inappropriate medication use or patient harm while the medication is in the control of the health care professional, patient or consumer. ${ }^{2}$ MEs are found at every stage of the medication process, with one-third of them being associated to medication preparation and administration procedures. ${ }^{3-5}$ On top of these alarming numbers, it is also known that only $2 \%$ of the MEs have been reported., ${ }^{4,6}$,

Medication administration is predominately the responsibility of the nursing staff and can be considered a nursing activity at high risk of errors. Many different factors contribute to MEs, including nurse's workload, time of duty shift, years of nursing experience, and system deficiencies. ${ }^{8,9}$ When nurses are surveyed, interruptions during medication administration appear to be one of the prominent causative factors of MEs. ${ }^{10-13}$

In the landmark report To err is human, the Institute of Medicine was among the first to suggest that interruptions could contribute to medical errors. ${ }^{14}$ In general, interruptions are recognized as conditions that reduce efficiency and productivity and contribute to errors in clinical practice. ${ }^{15}$ Westbrook and colleagues found that each interruption was associated with a $12.1 \%$ increase in procedural failures and a $12.7 \%$ increase in clinical errors. ${ }^{16}$ In a mixed methods study of the effect of interruptions on nurses' cognitive performance, medication preparation and administration procedures took up $16 \%$ of the nurses' working time. Furthermore, they also found that a high percentage of interruptions (22\%) occurred in medication rooms during preparation. ${ }^{17}$ These findings suggest that interventions are needed to eliminate or reduce interruptions that occur during the medication preparation and administration process. However, insights into the sources and characteristics of interruptions are needed before any intervention is designed. A systematic review by Biron and colleagues on the contribution of interruptions to medication administration errors found that studies should have demonstrated more methodological rigor through a more precise definition of interruptions and reliability reporting. ${ }^{18}$ Furthermore, most studies on interruptions are not specific to medication administration rounds (MARs), but are only applied to nurses' work in general or performed in intensive care units. Therefore, the evidence available so far on the characteristics of interruptions is limited to their frequencies from some important sources and is not specific to MARs in general nursing units. ${ }^{18} \mathrm{~A}$ better understanding of the characteristics and sources of interruptions during MARs would make it possible to tailor possible interventions in such a way that they most likely will
Correspondence: Marian Smeulers, Department Quality Assurance and Process Innovation (A3503), Academic Medical Center, Meibergdreef 9 , P.o.box 22600, 1100 DD Amsterdam, The Netherlands. Tel. +31.205668652

E-mail: m.smeulers@amc.uva.nl

Key words: work interruptions, medication administration errors, patient safety, nursing care, quality assurance.

Acknowledgments: the authors would like to thank the nursing units for their hospitality and for allowing us access as observers. The authors also would like to thank Nan van Geloven, PhD, for statistical advice.

Contributions: ED, F0, data collection and analyses; MS, analyses and manuscript writing; MH, manuscript reviewing; HV, study design and manuscript reviewing.

Conflict of interests: the authors declare no potential conflict of interests.

Funding: this study was funded by an internal AMC fund for quality improvement: Het zal je moeder maar wezen (What if your mother was in the hospital).

Received for publication: 17 January 2013.

Revision received: 22 April 2013

Accepted for publication: 22 April 2013.

This work is licensed under a Creative Commons Attribution NonCommercial 3.0 License (CC BYNC 3.0).

CC Copyright M. Smeulers et al., 2013

Licensee PAGEPress, Italy

Nursing Reports 2013; 3:e4

doi:10.4081/nursrep.2013.e4

have a significant impact on reducing interruptions and promote safe medication administration.

The aims of this study are: i) to examine the frequency and duration of interruptions and distractions during the daytime MARs (from medication preparation to administration) in the nursing units of an academic hospital; ii) to identify the causes of the interruptions and distractions; and iii) to compare any differences in the interruptions between surgical and non-surgical units.

\section{Materials and Methods}

\section{Setting}

The Academic Medical Center (AMC) is an 1024-bed tertiary care university teaching hospital in Amsterdam, The Netherlands. Medications at the study units were stored in a single medica- 
tion preparation room that was usually centrally located within the unit. Based on the physicians' prescriptions, nurses would prepare medications for their assigned patients either in medication rooms or in the corridor at standard or routine times (mainly at 8 a.m., 12 a.m., 6 p.m., and 10 p.m.). Preparation could involve collecting the medications from stock in the unit, checking them appropriately against the prescription sheets and mixing them for intravenous administration. After preparation, nurses would distribute and administer the medications to their assigned patients one by one.

\section{Study design}

Unobtrusive, structured observations of the MARs were used to obtain data on frequency and duration of the interruptions in the study units. ${ }^{19,20}$ The observed nurses were not aware of the actual specific aim of the observations in order not to influence their behavior and thus create performance bias. During the study briefing, the researchers stated that the medication administration process would be observed but gave no indication that the observations would be focused on interruptions. The nursing managers were informed of the purposes of the study and agreed to participate. Therefore, this study used unobtrusive observation of the behaviors of the nurses and their actions with their surrounding people and events during the MARs. ${ }^{21,22}$

\section{Sample and data collection procedure}

Since day shifts in clinical units are the most hectic and are most likely to be subject to interruptions and distractions, the decision was made to observe the MARs in the morning (8 a.m.) and at noon (12 a.m.). Based on similar sample sizes of the morning and noon MARs of two previous studies, we decided that the observation of 32 MARs would be sufficient for our study purposes. ${ }^{23,24}$ To be able to estimate inter-observer agreement during the study period, 2 observers measured the same MAR every two days. This added an extra seven observations for a total of 39 . The data collected for the purpose of inter-observer agreement could only be used once for the primary end points. One observation was randomly chosen by blinded draw for inclusion in the analysis. In each MAR, the duration of measurement was $60 \mathrm{~min}$ to ensure homogeneous results for better comparability between all observed MARs. Observations were performed on weekdays in a 2 -week period by 2 senior baccalaureate nursing students. The medication trolley was used as the site of measurement and as a consequence of this the observations were made in the medication room, as well as in the corridor. Whenever a nurse would enter a patient room with the medication, the observer would not follow but remain at the location of the medication trolley.

\section{Definitions and evaluation criteria}

An interruption or a distraction was defined as both an event initiated by another professional(s) or something else, and when a nurse interrupted him- or herself. In this review, the term interruption will be used for distractions as well as for interruptions. In addition, the kind of interruptions nurses experience was of interest. Again, based on the same previously published studies, data were collected on eight different categories of interruptions. ${ }^{23,24}$ These categories of interruptions were grouped into either verbal or non-verbal interruptions, resulting in the following categories: verbal or non-verbal interaction with a colleague, verbal or non-verbal interaction with a patient, verbal or non-verbal interaction with another person, non-verbal interaction with the environment, non-verbal logistical events. Table 1 shows how each category is defined. A paper-based data collection form was developed to record interruption characteristics: source of the interruption, duration of the interruption in seconds, location of the interruption (medication room or corridor), and category of the interruption. Other collected data were: morning or noon round, surgical or non-surgical unit. Data concerning the gender and level of training of the nursing staff (registered nurse, RN) were also collected. The data collection form was validated by a nested inter-observer agreement study of the 2 observers. The inter-class correlation coefficient (ICC) was used to determine interobserver agreement. Inter-observer agreement concerns whether or not an interruption occurs at a certain time and how long it lasts. A two-way fixed model was used to determine the inter-class correlation for each category of interruption. For the number of interruptions for each of the eight categories, six categories scored an ICC higher than 0.9 , one category scored 0.5 (non-verbal colleague), and one category scored 0.8 (verbal patient). For the dura- tion of the interruptions, all eight categories scored an ICC higher than 0.9. Therefore it was concluded that there was high interobserver agreement.

\section{Data analysis}

Data were entered into an MS-Excel spreadsheet by 2 observers (ED and FO) and crosschecked. The spreadsheet data were checked with the original data formed the third time by a third researcher (MS) using SPSS 16.0 software (IBM Corp., Armonk, NY, USA). Descriptive statistics were used to calculate the number and duration of the interruptions. Due to a skewed distribution of the data, the medians and interquartile ranges (IQR) were used to describe the spread of the data set. The IQR represented the difference between the first and third quartile of each set of data. Nonparametric Mann-Whitney U tests were used to compare the interruptions between the surgical and non-surgical units, and between the morning and noon MARs.

\section{Ethical approval}

Ethical approval was not considered necessary by The Institutional Review Board of the Academic Medical Centre at the University of Amsterdam, according to the Dutch Medical Ethics Law (i.e., a letter of exemption had been received from the Review Board could be requested from the authors).

\section{Results}

A total of 39 medication rounds were observed over a 2 -week period during which 32 observations were included for analysis (16 morning and 16 noon rounds, 15 non-surgical and 17 surgical MARs). Ten different nursing

Table 1. Categories of interruptions.

\begin{tabular}{|c|c|}
\hline Category & Description \\
\hline Verbal colleague & Colleague initiates a dialog with nurse preparing/administrating \\
\hline Verbal person & Nurse preparing/administrating initiates a dialog \\
\hline Verbal patient & Patient initiates a dialog with nurse preparing/administrating \\
\hline Non-verbal colleague & $\begin{array}{l}\text { Colleagues initiates a non-verbal interruption, e.g. getting supplies } \\
\text { in the vicinity of the nurse who is preparing/administrating }\end{array}$ \\
\hline Non-verbal person & $\begin{array}{l}\text { Nurse preparing/administrating initiates an interruption, } \\
\text { e.g. by helping a colleague or responding to a pager }\end{array}$ \\
\hline Non-verbal patient & $\begin{array}{l}\text { Patient initiates non-verbal interruption, e.g. by coming into the vicinity } \\
\text { of the nurse preparing/administrating }\end{array}$ \\
\hline Non-verbal surrounding & $\begin{array}{l}\text { Surrounding environment of the nurse initiates interruption, } \\
\text { e.g. noises from pagers, conversations in the vicinity of the nurse, } \\
\text { cleaners or stock management by pharmacy staff }\end{array}$ \\
\hline Non-verbal logistics & $\begin{array}{l}\text { Missing supplies for preparing the medications initiate an interruption } \\
\text { in the activities of the nurse preparing/administrating }\end{array}$ \\
\hline
\end{tabular}


units were observed (Table 2) for a total of 172 nurses. Participants were mostly female $(\mathrm{n}=144)$ and two-thirds of the nurses were RN level four or five $(n=118)$. In most of the cases medication was prepared in the medication room, resulting in 19 observations in that setting, in four cases medications were prepared in the corridor, and a combination of preparing in the corridor and in the medication room was observed nine times.

\section{Interruption rate}

In total, nurses were interrupted 1180 times during the observation period, for a rate of 6.9 interruptions per nurse per hour. This was divided in a total of 578 verbal interruptions (3.4 per nurse per hour) and a total of 602 nonverbal interruptions (3.5 per nurse per hour). Table 3 shows the basic interruption findings, classified by type of source. Most of the interruptions were caused by colleagues with a mean frequency of 9.0 (IQR 6.0-14.0) verbal interruptions and 5.0 (IQR 2.0-8.5) non-verbal interruptions, and noises from the surrounding environment with a mean frequency of 7.5 (IQR 4.0-12.8). Remarkably, only $3 \%$ of the interruptions were caused by patients (verbal $2 \%$ and non-verbal $1 \%)$.

\section{Duration of interruptions}

The total duration of the interruptions was $553.8 \mathrm{~min}$ (Table 3), a rate of $17.3 \mathrm{~min}$ per hour. This was divided in a total duration of $209.1 \mathrm{~min}$ for verbal interruptions and 344.7 min for the non-verbal interruptions. The longest interruptions are the verbal interruptions by colleagues with a median duration of 3.2 min (IQR 1.5-4.9). Non-verbal interruptions by colleagues have a median duration of 1.9 min (IQR 0.7-5.0). The second longest interruptions were caused by the non-verbal surrounding environment with a median duration of 2.9 min (IQR 0.8-5.0).

\section{Comparison of interruptions between surgical and non-surgical units}

A comparison between the units with surgical patients and the units with non-surgical patients (Table 4) reveals that more interruptions occurred in the non-surgical units (median 39.0; IQR 20.0-54.0) compared to the surgical units (median 29.0; IQR 23.0-42.5). Verbal interruptions occurred more often in surgical units and non-verbal interruptions in non-surgical units. The Mann-Whitney U test showed no statistically significant differences between the surgical and non-surgical units (Table 4).

\section{Comparison of interruptions between morning and noon medication administration rounds}

Comparing the morning MAR to the noon MAR showed that more interruptions were observed during the morning round (median 46.0; IQR 27.3-53.8) than during the noon round (median 28.0; IQR 17.0-37.5) (Table 5). The difference proved statistically significant for the verbal interruptions $(\mathrm{P}=0.047)$, the non-verbal interruptions $(\mathrm{P}=0.035)$, and for the total of the interruptions $(\mathrm{P}=0.005)$. The durations of the interruptions were longer during the morning rounds with a median duration of 21.5 min (IQR 12.8-27.9), compared with a median duration of $12.5 \mathrm{~min}$ (IQR 9.0 18.0) during the noon rounds. The difference was statistically significant for the non-verbal interruptions $(\mathrm{P}=0.029)$, as well as for the total duration $(\mathrm{P}=0.032)$.

\section{Discussion}

Emerging research demonstrates that interruptions are a risk for errors in medication administration. ${ }^{16,25}$ Therefore, we set out to gain a broad understanding of the possible causes for interruptions. The data obtained varied from direct interruptions leading to nurses having to perform other tasks to indirect peripheral distractions in the surrounding environment. Nurses are frequently disturbed during the MAR in their day shifts, which is consistent with other literature. The interruption rate of 6.9 interruptions per nurse each hour is similar to that reported in two studies by Biron et al. (6.3 and 6.7 interruptions per hour, respectively). ${ }^{18,26}$ Approximately onethird of the observed time is spent dealing with interruptions, indicating that it is a significant issue in nursing practice. The data also suggest that there may be a relationship between time of day and the number and duration of interruptions, with more and longer interruptions in the morning MAR. This is also consistent with other studies. ${ }^{18,24}$ This may be due to the general commotion of starting the day, as well as a desire to share events of one's per-

Table 2. Number of observations per nursing unit.

Unit Observations

\section{Surgical}

General surgery 4

General surgery and maxillary surgery 4

Cardiothoracic surgery 2

Orthopedics 4

Gynecology

3

Non-surgical

Cardiology

Infectious diseases 2

Internal medicine and kidney diseases 3

Gastroenterology and rheumatology 4

Acute renal dialysis 3

Total

32

Table 3. Basic interruption data: number and duration of interruptions.

\begin{tabular}{|c|c|c|c|c|}
\hline & No. of i & $=32)$ & Duration & ons $(n=32)$ \\
\hline & Frequency (\%) & Median (IQR) & Duration* (\%) & Median (IQR) \\
\hline Verbal & $578(49)$ & $17.0(11.0-23.3)$ & $209.1(38)$ & $6.4(3.6-8.1)$ \\
\hline Verbal colleague & $336(28)$ & $9.0(6.0-14.0)$ & $112.7(20)$ & $3.2(1.5-4.9)$ \\
\hline Verbal patient & $21(2)$ & $0.0(0.0-1.0)$ & $13.7(3)$ & $0.0(0.0-0.3)$ \\
\hline Verbal person & $221(19)$ & $5.0(3.3-8.8)$ & $82.7(15)$ & $1.9(1.0-3.5)$ \\
\hline Non-verbal & $602(51)$ & $15.0(11.0-26.5)$ & $344.7(62)$ & $10.0(5.1-16.1)$ \\
\hline Non-verbal colleague & $181(15)$ & $5.0(2.0-8.5)$ & $100.8(18)$ & $1.9(0.7-5.0)$ \\
\hline Non-verbal patient & $11(1)$ & $0.0(0.0-1.0)$ & $11.0(2)$ & $0.0(0.0-0.1)$ \\
\hline Non-verbal person & $35(3)$ & $1.0(0.0-2.0)$ & $38.2(7)$ & $0.5(0.0-1.7)$ \\
\hline Non-verbal surrounding environment & $289(25)$ & $7.5(4.0-12.8)$ & $106.8(19)$ & $2.9(0.8-5.0)$ \\
\hline Non-verbal logistics & $86(7)$ & $2.0(1.0-4.0)$ & $87.9(16)$ & $0.6(0.1-2.5)$ \\
\hline Total & $1180(100)$ & $34.0(21.5-51.5)$ & $553.8(100)$ & $14.0(9.6-25.2)$ \\
\hline
\end{tabular}

IQR, interquartile ranges. ${ }^{*}$ In minutes. 
sonal life on arriving at work. The data suggest that interruptions seemed to occur more often and lasted longer in units with non-surgical patients compared to units with surgical patients. However, the observed difference was not statistically significant. Another study by McGillis et al. also compared interruptions between surgical and non-surgical units and had similar findings. ${ }^{27}$ In addition, our study was limited in time and measurements, and did not reveal any specific clues regarding the causes of medication errors. Possibly differences in the culture of the nursing unit, the type of patients cared for, or differences in the way nursing tasks were organized in these units might be the reason for this.

Verbal and non-verbal interruptions caused by colleagues appear to be a significant issue. Almost half of the observed causes of interruptions are verbal and non-verbal interruptions from colleagues. Other studies also confirm colleagues to be the main source of interruptions. ${ }^{18,23,24}$ Targeting interruptions by colleagues seems an obvious way to improve medication safety. In recent years, several interventions have been introduced to reduce the interruptions of medication administration, varying from visible reminders such as do-notdisturb tabards and signs to remind others of no interruption during MARs, to dedicated quiet zones, protocols and educational strategies. ${ }^{15,25,28-32}$ Results are promising, with most of these quality improvement studies reporting positive effects on the number of interruptions. However, implementing such interventions may be difficult. Nurses may find it difficult to call each other to account when they are in the middle of an important task. Also wearing do-not-disturb tabards, which is currently a popular intervention to target interruptions, may meet with resistance (M Smeulers, unpublished data, 2012). To address this, behavioral or cultural interventions targeting the way nurses interact or perceive each other may also be needed. ${ }^{33,34}$

This study also revealed that interruptions from the surrounding environment are an important factor to target when designing interventions that aim to reduce interruptions. ${ }^{28}$ Changing procedures in the medication room, such as cleaning and re-stocking times, or adapting the lay-out may be possible measures to take. ${ }^{35}$

Since interruptions originate from a variety of many different sources, a multi-faceted approach that targets not only a decrease in interruptions by colleagues but also considers ways to decrease interruptions from the surrounding environment seems appropriate. Furthermore, increasing the awareness of the nurses about being disturbed might be useful to lower self-initiated interruptions and conversations on subjects other than patient care

Table 4. Comparison of number and duration of interruptions in surgical and non-surgical units.

\begin{tabular}{|c|c|c|c|c|c|c|c|c|c|c|}
\hline \multirow[b]{2}{*}{ Interruptions } & \multicolumn{4}{|c|}{ Surgical (n=17) } & \multicolumn{4}{|c|}{ Non-surgical ( $\mathrm{n}=15)$} & \multicolumn{2}{|c|}{ Mann-Whitney U Test } \\
\hline & $\begin{array}{l}\text { Frequency } \\
\qquad(\%)\end{array}$ & $\begin{array}{l}\text { Median } \\
\text { (IQR) }\end{array}$ & $\begin{array}{l}\text { Duration* } \\
\text { (\%) }\end{array}$ & $\begin{array}{l}\text { Median } \\
\text { (IQR) }\end{array}$ & $\begin{array}{l}\text { Frequency } \\
\qquad(\%)\end{array}$ & $\begin{array}{l}\text { Median } \\
\text { (IQR) }\end{array}$ & $\begin{array}{c}\text { Duration* } \\
(\%)\end{array}$ & $\begin{array}{l}\text { Median } \\
\text { (IQR) }\end{array}$ & $\begin{array}{l}\text { Frequency } \\
\text { (P-value) }\end{array}$ & $\begin{array}{l}\text { Duration } \\
\text { (P-value) }\end{array}$ \\
\hline Verbal & $296(52)$ & $17.0(12.0-20.5)$ & $100.6(34)$ & $5.6(3.8-7.3)$ & $282(46)$ & $13.0(6.0-27.0)$ & $108.6(38)$ & $7.1(3.0-8.9)$ & 0.794 & 0.576 \\
\hline Verbal colleague & $181(61)$ & $9.0(7.0-13.5)$ & $54.7(54)$ & $3.1(1.6-4.2)$ & $155(55)$ & $9.0(3.0-15.0)$ & $58.1(53)$ & $3.6(1.5-5.9)$ & 0.823 & 0.602 \\
\hline Verbal patient & $19(6)$ & $0.0(0.0-2.0)$ & $9.6(10)$ & $0.0(0.0-1.1)$ & $2(1)$ & $0.0(0.0-0.0)$ & $4.1(4)$ & $0.0(0.0-0.0)$ & 0.132 & 0.176 \\
\hline Verbal person & $96(33)$ & $5.0(3.5-8.0)$ & $36.3(36)$ & $1.9(1.1-2.5)$ & $125(44)$ & $4.0(3.0-13.0)$ & $46.4(43)$ & $3.0(1.0-4.2)$ & 0.628 & 0.433 \\
\hline Non-verbal & $274(52)$ & $13.0(9.0-22.5)$ & $168.5(57)$ & $7.3(4.6-16.0)$ & $328(54)$ & $16.0(13.0-30.0)$ & $176.2(62)$ & $10.6(5.0-18.4)$ & 0.097 & 0.628 \\
\hline Non-verbal colleague & $90(30)$ & $3.0(2.0-7.0)$ & $52.4(31)$ & $1.9(0.7-5.4)$ & $91(28)$ & $5.0(3.0-10.0)$ & $48.5(28)$ & $3.0(0.7-5.0)$ & 0.295 & 0.941 \\
\hline Non-verbal patient & $8(3)$ & $0.0(0.0-1.0)$ & $6.8(4)$ & $0.0(0.0-0.6)$ & $3(1)$ & $0.0(0.0-0.0)$ & $4.2(2)$ & $0.0(0.0-0.0)$ & 0.433 & 0.370 \\
\hline Non-verbal person & $20(7)$ & $1.0(0.0-2.0)$ & $22.3(13)$ & $0.3(0.0-1.7)$ & $15(4)$ & $1.0(0.0-2.0)$ & $15.9(9)$ & $0.7(0.0-2.0)$ & 0.710 & 0.941 \\
\hline $\begin{array}{l}\text { Non-verbal surrounding } \\
\text { environment }\end{array}$ & $125(42)$ & $7.0(3.0-11.0)$ & $58.9(35)$ & $2.8(0.8-5.3)$ & $164(50)$ & $9.0(4.0-18.0)$ & $47.9(27)$ & $3.7(0.8-4.9)$ & 0.189 & 0.852 \\
\hline Non-verbal logistics & $31(10)$ & $1.0(0.5-2.5)$ & $28.1(17)$ & $0.4(0.1-1.4)$ & $55(17)$ & $3.0(1.0-5.0)$ & $59.8(34)$ & $1.0(0.3-5.6)$ & 0.053 & 0.165 \\
\hline Total & $570(48)$ & $29.0(23.0-42.5)$ & $269.1(49)$ & $13.7(9.4-21.5)$ & $610(52)$ & $39.0(20.0-54.0)$ & $284.7(51)$ & $14.2(9.8-27.0)$ & 0.390 & 0.433 \\
\hline
\end{tabular}

IQR, interquartile ranges. *In minutes.

Table 5. Comparison of number and duration of interruptions between morning and noon medication administration rounds.

\begin{tabular}{|c|c|c|c|c|c|c|c|c|c|c|}
\hline \multirow[b]{2}{*}{ Interruptions } & \multirow[b]{2}{*}{$\begin{array}{c}\text { Frequency } \\
\qquad(\%)\end{array}$} & \multicolumn{3}{|c|}{ Morning MAR (n-16) } & \multicolumn{4}{|c|}{ Noon MAR ( $\mathrm{n}=16)$} & \multicolumn{2}{|c|}{ Mann-Whitney U Test } \\
\hline & & $\begin{array}{l}\text { Median } \\
\text { (IQR) }\end{array}$ & $\begin{array}{c}\text { Duration* } \\
(\%)\end{array}$ & $\begin{array}{l}\text { Median } \\
\text { (IQR) }\end{array}$ & $\begin{array}{c}\text { Frequency } \\
\qquad(\%)\end{array}$ & $\begin{array}{l}\text { Median } \\
\text { (IQR) }\end{array}$ & $\begin{array}{c}\text { Duration* } \\
(\%)\end{array}$ & $\begin{array}{c}\text { Median } \\
\text { (IQR) }\end{array}$ & $\begin{array}{l}\text { Frequency } \\
\text { (P-value) }\end{array}$ & $\begin{array}{l}\text { Duration } \\
\text { (P-value) }\end{array}$ \\
\hline Verbal & $353(48)$ & $19.0(12.3-31.0)$ & $119.8(35)$ & $6.7(3.8-8.9)$ & $225(50)$ & $13.5(6.8-20.5)$ & $89.3(42)$ & $5.5(3.1-7.8)$ & $0.047^{\circ}$ & 0.323 \\
\hline Verbal colleague & $200(27)$ & $9.5(8-17.3)$ & $59.1(17)$ & $3.0(1.4-5.3)$ & $136(30)$ & $8.5(3.3-13.0)$ & $53.7(25)$ & $3.4(1.9-4.8)$ & 0.094 & 0.985 \\
\hline Verbal patient & $11(2)$ & $0.0(0.0-0.8)$ & $7.6(2)$ & $0.0(0.0-0.8)$ & $10(2)$ & $0.0(0.0-1.0)$ & $6.1(3)$ & $0.0(0.0-0.3)$ & 0.867 & 0.897 \\
\hline Verbal person & $142(20)$ & $7.0(4.3-12.3)$ & $53.1(16)$ & $2.1(1.4-4.1)$ & $79(17)$ & $4.0(3.0-7.8)$ & $29.6(14)$ & $1.7(0.6-2.9)$ & $0.035^{\circ}$ & 0.119 \\
\hline Non-verbal & $375(52)$ & $24.5(12.0-33.3)$ & $219.4(65)$ & $14.4(5.6-19.8)$ & $227(50)$ & $13.0(11.0-15.8)$ & $125.3(58)$ & $6.0(3.5-10.6)$ & $0.035^{\circ}$ & $0.029^{\circ}$ \\
\hline Non-verbal colleague & $116(16)$ & $6.0(2.3-11.5)$ & $67.5(20)$ & $4.1(0.9-6.3)$ & $65(14)$ & $4.0(2.0-5.0)$ & $33.3(15)$ & $1.2(0.7-2.0)$ & 0.128 & 0.073 \\
\hline Non-verbal patient & $7(1)$ & $0.0(0.0-1.0)$ & $9.6(3)$ & $0.0(0.0-1.1)$ & $4(1)$ & $0.0(0.0-0.8)$ & $1.4(1)$ & $0.0(0.0-0.0)$ & 0.724 & 0.402 \\
\hline Non-verbal person & $19(3)$ & $1.0(0.0-2.0)$ & $23.5(7)$ & $0.7(0.0-2.2)$ & $16(4)$ & $1.0(0.0-1.8)$ & $14.8(7)$ & $0.2(0.0-1.6)$ & 0.669 & 0.669 \\
\hline $\begin{array}{l}\text { Non-verbal surrounding } \\
\text { environment }\end{array}$ & $186(26)$ & $11.0(7.0-16.5)$ & $74.3(22)$ & $4.6(3.0-5.6)$ & $103(23)$ & $4.5(2.3-8.8)$ & $32.5(15)$ & $1.0(0.5-2.9)$ & $0.007^{\circ}$ & $0.007^{\circ}$ \\
\hline Non-verbal logistics & $47(6)$ & $2.0(0.0-4.0)$ & $44.6(13)$ & $0.5(0.0-4.3)$ & $39(9)$ & $2.0(1.0-3.0)$ & $43.3(20)$ & $0.7(0.2-2.1)$ & 0.696 & 0.539 \\
\hline Total & $728(62)$ & $46.0(27.3-53.8)$ & $339.1(61)$ & $21.5(12.8-27.9)$ & $452(38)$ & $28.0(17.0-37.5)$ & $214.7(39)$ & $12.5(9.0-18.0)$ & $0.005^{\circ}$ & $0.032^{\circ}$ \\
\hline
\end{tabular}

MAR, medication administration rounds; IQR, interquartile ranges. ${ }^{*}$ In minutes; ${ }^{\circ}$ Significant $(\mathrm{P}<0.05)$. IQR, interquartile ranges. ${ }^{*}$ In minutes. 
(sterile cockpit rule from aviation profession). ${ }^{30,34}$ Performing observational studies such as those carried out by the researchers may serve as an eye-opener and contribute to a culture of continuous improvement. Last but not least, no matter how evident the need is to limit the number of interruptions, the ultimate goal is to decrease the number of MEs. So far the empirical evidence of an association between interruptions en MEs, however promising, is still limited and more research into this area is also needed. ${ }^{16,30,36}$

\section{Study limitations}

Our study has several limitations. The study was performed on surgical and non-surgical units of one academic hospital. Pediatric and neurological units were not included, and only the morning and noon MARs during the week were observed in a limited time period. Therefore, generalizability of the results may be limited, especially to other MAR times and during weekends. One remarkable finding was that there are seldom any interruptions by patients, whereas usually staff perceptions are that these occur much more often than they actually do. However, the observations of the interruptions were limited to the medication trolley. As a consequence, the observer did not follow the nurse into the patients' rooms. Therefore, the number of interruptions by patients may be underestimated. Due to the nature of the study, observations could not be performed blinded. However, the observations were unobtrusive and the subjects were not aware of the specific purpose of the study.

Future research should consider including all MAR times (day and night time as well as weekends) and record observations for an extended period of time. Establishing a clear definition of interruptions, as well as testing inter-observer reliability prior to the observation period, proved to be valuable. Including other specialties, and looking into possible differences between them, may provide evidence for a significant difference between the specialties and possible causes for this.

In a follow-up study, the effect of the introduction of do-not-disturb tabards on the frequency of interruptions (verbal as well as nonverbal) and error rate during the MAR will be determined, as well as the relationship between interruptions and MEs.

\section{Implications and future research}

Interruptions are an important problem in the complex reality of nursing practice because of their high frequency and for the risk they introduce into medication process. Interruptions come from many different sources: colleagues, the surrounding environment or the nurse him- or herself. An ideal mix of interventions targets not only a reduction in interruptions by colleagues, but also considers ways to decrease self-initiated interruptions and reduce sources of interruptions in the nurse's surrounding environment. Finally, understanding the causality between interruptions and MEs is an important topic for further research.

\section{References}

1. Committee on Identifying and Preventing Medication Errors. Preventing medication errors: quality chasm series. Washington, DC: The National Academies Press; 2007.

2. NCC MERP. National Coordinating Council for Medication Error Reporting and Prevention. What is a medication error?; 2012. Available from: http://www.nccmerp. org/aboutMedErrors.html

3. Fijn R, van den Bemt PM, Chow M, et al. Hospital prescribing errors: epidemiological assessment of predictors. Br J Clin Pharmacol 2002;53:326-31.

4. Barker KN, Flynn EA, Pepper GA. Observation method of detecting medication errors. Am J Health Syst Pharm 2002;59:2314-6.

5. Leape LL, Bates DW, Cullen DJ, et al. Systems analysis of adverse drug events. ADE Prevention Study Group. JAMA 1995;274:35-43.

6. Gladstone J. Drug administration errors: a study into the factors underlying the occurrence and reporting of drug errors in a district general hospital. J Adv Nurs 1995;22:628-37.

7. Antonow JA, Smith AB, Silver MP. Medication error reporting: a survey of nursing staff. J Nurs Care Qual 2000;15: 42-8.

8. Armitage G, Knapman H. Adverse events in drug administration: a literature review. $\mathrm{J}$ Nurs Manag 2003;11:130-40.

9. Tang FI, Sheu SJ, Yu S, et al. Nurses relate the contributing factors involved in medication errors. J Clin Nurs 2007;16:447-57.

10. Armutlu M, Foley ML, Surette J, et al. Survey of nursing perceptions of medication administration practices, perceived sources of errors and reporting behaviours. Healthc Q 2008;11:58-65.

11. Balas MC, Scott LD, Rogers AE. The prevalence and nature of errors and near errors reported by hospital staff nurses. Appl Nurs Res 2004;17:224-30.

12. Mayo AM, Duncan D. Nurse perceptions of medication errors: what we need to know for patient safety. J Nurs Care Qual 2004;19:209-17.

13. Stratton KM, Blegen MA, Pepper G, Vaughn T. Reporting of medication errors by pediatric nurses. J Pediatr Nurs 2004;19:385-92.
14. Committee on Quality of Health Care in America, Institute of Medicine. To err is human: Building a safer health system. Washington, DC: The National Academies Press; 2000.

15. Pape TM. Applying airline safety practices to medication administration. Medsurg Nurs 2003;12:77-93.

16. Westbrook JI, Woods A, Rob MI, et al. Association of interruptions with an increased risk and severity of medication administration errors. Arch Intern Med 2010;26:683-90.

17. Potter P, Wolf L, Boxerman S, et al. An analysis of nurses' cognitive work: a new perspective for understanding medical errors. Rockville, MC: The Agency for Healthcare Research and Quality; 2005.

18. Biron AD, Loiselle CG, Lavoie-Tremblay M. Work interruptions and their contribution to medication administration errors: an evidence review. Worldviews Evid Based Nurs 2009;6:70-86.

19. Flynn EA, Barker KN, Pepper GA, et al. Comparison of methods for detecting medication errors in 36 hospitals and skillednursing facilities. Am J Health Syst Pharm 2002;59:436-46.

20. Meyer-Massetti C, Cheng CM, Schwappach DL, et al. Systematic review of medication safety assessment methods. Am J Health Syst Pharm 2011;68:227-40.

21. Berdot S, Sabatier B, Gillaizeau F, et al. Evaluation of drug administration errors in a teaching hospital. BMC Health Serv Rev 2012;12:60.

22. Rodriguez-Gonzalez CG, Herranz-Alonso A, Martin-Barbero ML, et al. Prevalence of medication administration errors in two medical units with automated prescription and dispensing. J Am Med Inform Assoc 2012;19:72-8.

23. Kreckler S, Catchpole K, Bottomley M, et al. Interruptions during drug rounds: an observational study. Br J Nurs 2008;17: 1326-30.

24. Palese A, Sartor A, Costaperaria G, Bresadola V. Interruptions during nurses' drug rounds in surgical wards: observational study. J Nurs Manag 2009;17:185-92.

25. Pape TM, Guerra DM, Muzquiz M, et al. Innovative approaches to reducing nurses' distractions during medication administration. J Contin Educ Nurs 2005;36:108-16.

26. Biron AD, Lavoie-Tremblay M, Loiselle CG. Characteristics of work interruptions during medication administration. J Nurs Scholarsh 2009;41:330-6.

27. McGillis HL, Pedersen C, Fairley L. Losing the moment: understanding interruptions to nurses' work. J Nurs Adm 2010;40: 169-76.

28. Relihan E, O’Brien V, O'Hara S, Silke B. The impact of a set of interventions to 
reduce interruptions and distractions to nurses during medication administration. Qual Saf Health Care 2010;19:e52.

29. Tomietto M, Sartor A, Mazzocoli E, Palese A. Paradoxical effects of a hospital-based, multi-intervention programme aimed at reducing medication round interruptions. J Nurs Manag 2012;20:335-43.

30. Fore AM, Sculli GL, Albee D, Neily J. Improving patient safety using the sterile cockpit principle during medication administration: a collaborative, unit-based project. J Nurs Manag 2013;21:106-11.

31. Scott J, Williams D, Ingram J, Mackenzie F. The effectiveness of drug round tabards in reducing incidence of medication errors. Nurs Times 2010;106:13-5.

32. Anthony K, Wiencek C, Bauer C, et al. No interruptions please: impact of a No Interruption Zone on medication safety in intensive care units. Crit Care Nurse 2010;30:21-9.

33. Julianne M. Nurses create a culture of patient safety: it takes more than projects.
Online J Issues Nurs 2011;16:2.

34. Walrath JM, Dang D, Nyberg D. An organizational assessment of disruptive clinician behavior: findings and implications. J Nurs Care Qual 2013;28:110-21.

35. Conrad C, Fields W, McNamara T, et al. Medication room madness: calming the chaos. J Nurs Care Qual 2010;25:137-44.

36. Grundgeiger T, Sanderson P. Interruptions in healthcare: theoretical views. Int J Med Inform 2009;78:293-307. 